\title{
Artificial Intelligence-Enhanced Smartwatch ECG for Heart Failure-Reduced Ejection Fraction Detection by Generating 12-Lead ECG
}

\author{
Joon-myoung Kwon ${ }^{1,2,3,4,+} \mathbb{D}$, Yong-Yeon Jo ${ }^{1,+} \mathbb{D}$, Soo Youn Lee ${ }^{2,5}$, Seonmi Kang ${ }^{1}$, Seon-Yu Lim ${ }^{1}$, \\ Min Sung Lee ${ }^{1,2,3}$ and Kyung-Hee Kim ${ }^{2,5, * \mathbb{D}}$
}

check for

updates

Citation: Kwon, J.-m.; Jo, Y.-Y.; Lee, S.Y.; Kang, S.; Lim, S.-Y.; Lee, M.S.; Kim, K.-H. Artificial IntelligenceEnhanced Smartwatch ECG for Heart Failure-Reduced Ejection Fraction Detection by Generating 12-Lead ECG. Diagnostics 2022, 12, 654. https:// doi.org/10.3390/diagnostics12030654

Academic Editors: Keun Ho Ryu and Nipon Theera-Umpon

Received: 1 February 2022

Accepted: 2 March 2022

Published: 8 March 2022

Publisher's Note: MDPI stays neutral with regard to jurisdictional claims in published maps and institutional affiliations.

Copyright: (C) 2022 by the authors. Licensee MDPI, Basel, Switzerland. This article is an open access article distributed under the terms and conditions of the Creative Commons Attribution (CC BY) license (https:// creativecommons.org/licenses/by/ $4.0 /)$.
1 Medical Research Team, Medical AI, Inc., San Francisco, CA 94103, USA; happywithhj@gmail.com (J.-m.K.); yy.jo@medicalai.com (Y.-Y.J.); seonmikang@medicalai.com (S.K.); imsun211@medicalai.com (S.-Y.L.); lylm@medicalai.com (M.S.L.)

2 Artificial Intelligence and Big Data Research Center, Sejong Medical Research Institute, Bucheon 14754, Korea; leesy@sejongh.co.kr

3 Department of Critical Care and Emergency Medicine, Incheon Sejong Hospital, Incheon 21080, Korea

4 Medical R\&D Center, Body Friend, Co., Ltd., Seoul 06302, Korea

5 Division of Cardiology, Cardiovascular Center, Incheon Sejong Hospital, Incheon 21080, Korea

* Correspondence: learnbyliving9@gmail.com; Tel.:+82-32-240-8568; Fax: +82-32-240-8094

+ These authors contributed equally to this work.

\begin{abstract}
Background: We developed and validated an artificial intelligence (AI)-enabled smartwatch ECG to detect heart failure-reduced ejection fraction (HFrEF). Methods: This was a cohort study involving two hospitals (A and B). We developed the AI in two steps. First, we developed an AI model (ECGT2T) to synthesize ten-lead ECG from the asynchronized 2-lead ECG (Lead I and II). ECGT2T is a deep learning model based on a generative adversarial network, which translates source ECGs to reference ECGs by learning styles of the reference ECGs. For this, we included adult patients aged $\geq 18$ years from hospital A with at least one digitally stored 12-lead ECG. Second, we developed an AI model to detect HFrEF using a 10 s 12-lead ECG. The AI model was based on convolutional neural network. For this, we included adult patients who underwent ECG and echocardiography within 14 days. To validate the AI, we included adult patients from hospital B who underwent two-lead smartwatch ECG and echocardiography on the same day. The AI model generates a $10 \mathrm{~s} 12$-lead ECG from a two-lead smartwatch ECG using ECGT2T and detects HFrEF using the generated 12-lead ECG. Results: We included 137,673 patients with 458,745 ECGs and 38,643 patients with 88,900 ECGs from hospital A for developing the ECGT2T and HFrEF detection models, respectively. The area under the receiver operating characteristic curve of AI for detecting HFrEF using smartwatch ECG was 0.934 (95\% confidence interval 0.913-0.955) with 755 patients from hospital B. The sensitivity, specificity, positive predictive value, and negative predictive value of AI were $0.897,0.860,0.258$, and 0.994, respectively. Conclusions: An AI-enabled smartwatch 2-lead ECG could detect HFrEF with reasonable performance.
\end{abstract}

Keywords: heart failure; electrocardiography; deep learning; artificial intelligence

\section{Introduction}

Heart failure (HF) is a significant healthcare burden worldwide, with an estimated 64.3 million people living with HF [1,2]. Despite advances in treatment, HF remains as a high risk of morbidity and mortality and is the most common diagnosis in hospitalized patients aged over 65 years, with a 5-year survival rate of only $57 \%$ [3-5]. In the United States, HF affects $\sim 30.7$ billion total annual costs and projection suggests that by 2030 , the total cost of HF will increase by $127 \%$, to $\$ 69.8$ billion $[3,6]$.

Patients suffering with HF with reduced ejection fraction (HFrEF) become less active, leading to repeated hospitalization, resulting in a poor quality of life, including a high 
medical cost burden [7]. Despite its poor prognosis and high economic burden, HFrEF awareness remains relatively low due to its insidious onset, varied presentation, and syndromic nature [8]. Early diagnosis and timely intervention may prevent irreversible HFrEF progression and mortality [9]. Electrocardiography (ECG) is a low-cost test frequently performed for a variety of purposes, especially basic examination and screening for cardiovascular disease [10]. We developed an artificial intelligence (AI)-enabled ECG algorithm, which can increase the diagnosis of HFrEF [11,12]. However, it is also inconvenient to visit the hospital for a 12-lead ECG.

Smartwatches have high processing power and sophisticated sensors that can provide new health data, including ECG. In this study, we developed and validated an artificial intelligence-enabled smartwatch ECG for HFrEF detection. As the smartwatch could obtain a single lead ECG, we also developed a deep learning-based model (DLM) for generating a 12-lead ECG from a smartwatch ECG (Lead I and II) to enhance AI performance and detect HFrEF using the generated 12-lead ECG. Moreover, we conducted internal and external validation for the developed $\mathrm{AI}$ in this multicenter study. To the best of our knowledge, this is the first study to generate a 12-lead ECG from a smartwatch ECG and detect heart failure. Based on this deep learning model, heart failure with reduced ejection fraction could be detected in daily living by using asynchronous 2-lead ECGs from lifestyle ECG devices, such as smart watches.

\section{Methods}

\subsection{Data Source and Study Population}

We conducted a multicenter retrospective cohort study to develop and validate the AI in two hospitals, as shown in Figure 1. Hospital A is a cardiovascular teaching hospital, and hospital B is a community general hospital. Data from hospital A were used for developing the AI and for internal performance tests, and data from hospital B for external performance tests with smartwatch ECG. First, we included all adult patients aged 18 years and older who underwent at least one $10 \mathrm{~s}$ 12-lead ECG at hospital A during the study period (1 November 2015-31 May 2021). The 10 s 12-lead ECG was acquired in the supine position and digitally stored at a $500 \mathrm{~Hz}$ sampling rate. The data were used to develop the AI models, with ECGT2T (ECG synthesis from two-lead to ten-lead) being used to generate the 12-lead ECG from two non-synchronized lead (Lead I and II) ECG. Second, we included adult patients aged 18 years and older who underwent both $10 \mathrm{~s}$ 12-lead ECG and echocardiography within 14 days at hospital A during the study period (1 November 2015-30 June 2021). The data were split into development data (80\%) to develop the AI model for detecting HFrEF using 12-lead ECG and internal performance test data (20\%). Third, for the external test dataset (smartwatch ECG), we included adult patients aged 18 years and older who underwent smartwatch ECG and echocardiography in the study period (1 June 2021-30 July 2021). We used two types of smartwatches-Galaxy Watch Active (Smart watch A) and Apple Watch 6 (Smart watch B). Two lead (Leads I and II) were obtained from each patient using each smartwatch. The method to obtain two 2-lead ECG using a smartwatch was described in a previous study [13]. The study population with missing clinical information, including ECG, echocardiographic results, or demographic data, was excluded. The Bucheon and Incheon Sejong Hospital Institutional Review Board approved this study protocol and waived the need for informed consent due to minimal harm and impracticality. This study complied with the Declaration of Helsinki. 


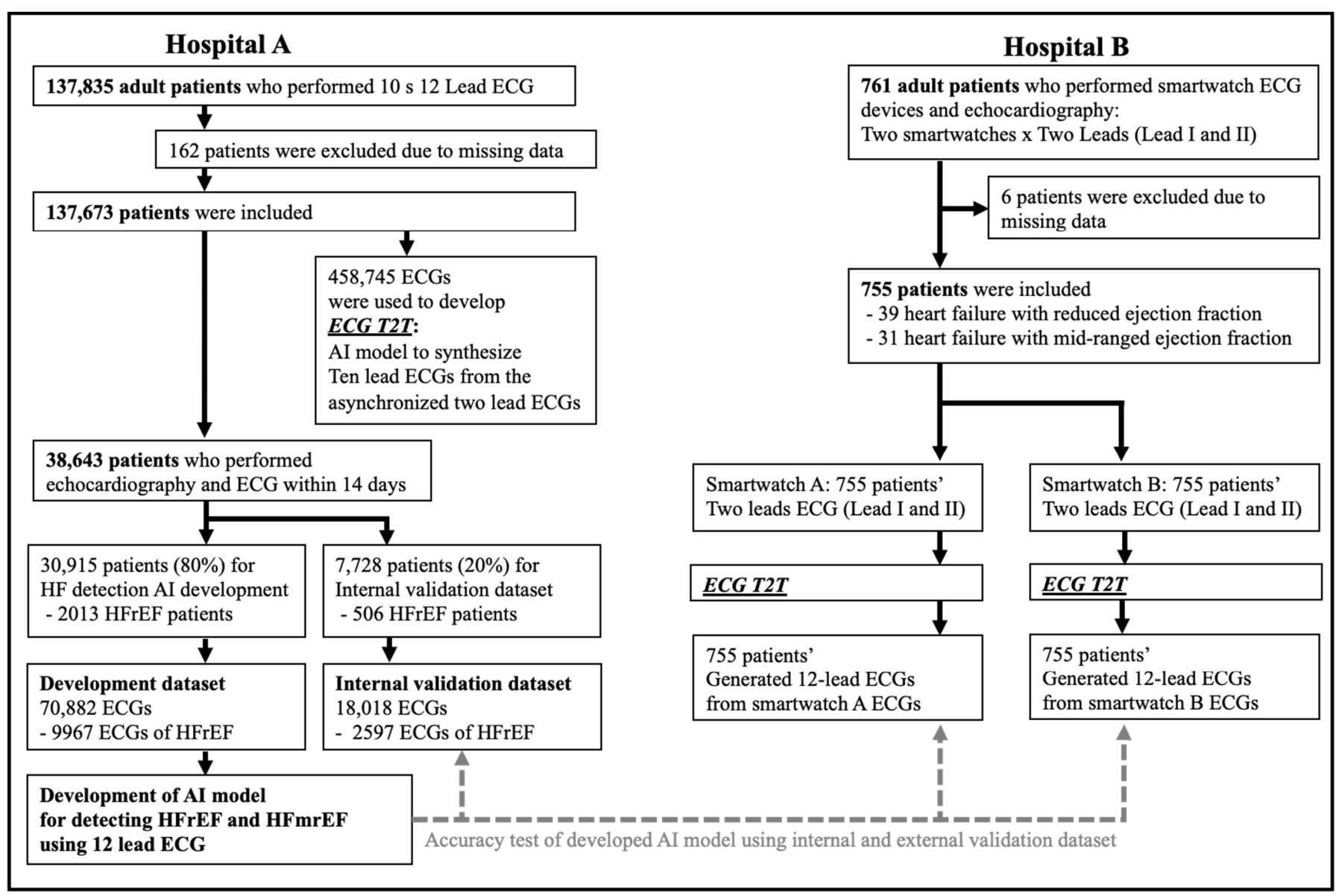

Figure 1. Study flowchart. Legend: AI denotes artificial intelligence, ECG electrocardiography, ECGT2T ECG synthesis from two-lead to ten-lead, HF heart failure, HFmrEF heart failure with mildly reduced ejection fraction, and HFrEF heart failure with reduced ejection fraction.

\subsection{Outcomes and Predictive Variables}

The primary outcome of this study was the performance of the AI in detecting patients with HFrEF using a standard $10 \mathrm{~s}$ 12-lead ECG or smartwatch ECG. HFrEF was defined as an ejection fraction of $40 \%$ or less on transthoracic comprehensive echocardiography, which was recorded in the electronic health record database at the time of acquisition, symptom, and signs from medical records [14]. The EF was determined using a biplane approach with the Simpson and 2D methods. If the estimated EF was in a range, we used the middle value as a single EF value. If more than two echocardiographies were obtained within 14 days from the ECG, we used echocardiography that was closest to ECG as index echocardiography. The secondary outcome was the performance of the AI in detecting patients with $\mathrm{HF}$ with mildly reduced $\mathrm{EF}(\mathrm{HFmrEF})$ to reduced $\mathrm{EF}(<50 \%)$ on echocardiography [14]. Predictive variables were ECG, age, sex, weight, and height.

\subsection{Data Preprocessing}

We preprocessed the ECGs for sampling, normalization, and augmentations. We constructed an ECG with $8 \mathrm{~s}$ by cropping of $1 \mathrm{~s}$ on each side and normalized (z-score) based on the mean and standard deviation. In terms of augmentations, the addition of linear and nonlinear noise causing baseline changes was performed. We also normalized the values of age, weight, and height. We changed the value of sex to one-hot encoding. 


\subsection{Development for a Platform Detecting HFrEF}

Our AI consists of two phases. First phase generates a standard 12-lead ECG. We developed an ECGT2T for generating a 12-lead ECG from an asynchronous 2-lead ECG, as shown in Figure 2. ECGT2T is a deep learning model based on a generative adversarial network that synthesizes a 10-lead ECG (III, aVR, aVL, aVF, and V1-6) from an asynchronous 2-lead ECG (leads I and II). It translates source ECGs to reference ECGs by learning the styles of reference ECGs; it first generates a single latent code representing the cardiac condition from two given leads and then reconstructs the other 10 leads by referring to the single latent code based on the single given lead. For ECGT2T development, development data from hospital A were used. We have described the detailed development process of ECGT2T in our previous article [15]

Second phase detects a HFrEF. We developed another deep learning model based on four residual blocks for detecting HFrEF using a 12-lead ECG. This model was trained using the development dataset from hospital A. Figure 2 show the architecture of the HFrEF detection model. Each residual block contains two submodules, each of which has a one-dimensional convolutional neural network, batch normalization, rectified linear unit activation, and a dropout layer. The difference between both sub modules is the skip connection. The first sub module has only the skip connection. The latent variables passing through all residual blocks are concatenated to auxiliary information including age, gender, height, and weight. Thereafter, the final output was obtained through two fully connected layers. We used the Adam optimizer, which is a popular algorithm in the field of deep learning because it achieves good results fast. Additionally, we found that its performance was better than that of SGD, RMSprop, or Adadelta in the pilot study. For parameter settings, we set the learning rate to $1 \times 10^{-4}$, weight decay to $1 \times 10^{-5}$, epsilon to $1 \times 10^{-8}$, beta1 to 0.9 , and beta 2 to 0.999 , respectively. We confirmed the values of parameters by grid search methods. We selected the search space of grid search by using random sampling search of pilot study. We train the models on a high-performance computer composed of 20 DGX servers with 160 NVIDIA A100 graphics processing units. All models were developed using PyTorch and Python.

\subsection{Statistical Analysis}

We used the area under the receiver operating characteristic curve (AUC) to confirm the performance based on the continuous prediction score and presence of $\mathrm{HFrEF}$. The $95 \%$ confidence intervals (CIs) of AUC were confirmed by the Sun and Su optimization of the DeLong method. We confirmed the sensitivity, specificity, positive predictive value (PPV), and negative predictive value (NPV) using a cut-off point. The cut-off point was defined using Youden's J statistics [16]. We used two-sided 95\% CIs to describe the variability of the study population and estimates. We used exact CIs based on Clopper-Pearson to be conservative for accuracy, sensitivity, specificity, PPV, and NPV. We analyzed the statistical results using $\mathrm{R}$ version 3.4.3.

\subsection{Role of the Funding Sources}

This study was supported by a National Research Foundation of Korea grant funded by the Korean government (No. 2020R1F1A1073791). None of the listed entities played any role in the design of the study; data collection, model development, result interpretation, writing article, or decision to submit this paper. All authors had full access to the data and the final decision of submission. 


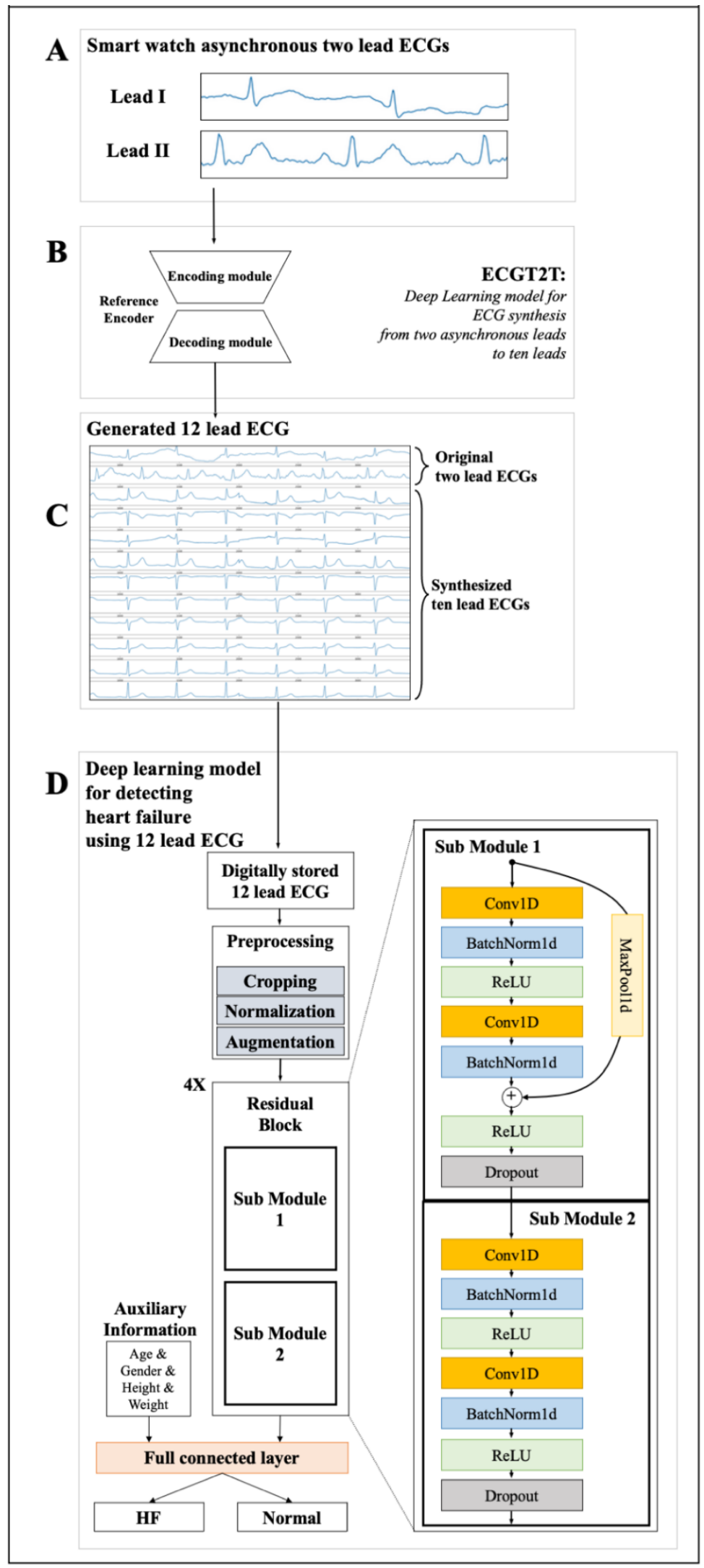

Figure 2. Architecture of deep learning model for detecting heart failure. Legend: ECG denotes electrocardiography, ECGT2T ECG synthesis from two-lead to ten-lead, and HF heart failure. (A) Asynchronous two lead ECGs from smart watch. (B) ECGT2T for synthesizing ten lead ECG from two lead ECG. (C) Generated twelve lead ECG which input to final AI model. (D) Deep learning model for detecting heart failure with reduced ejection fraction using generated twelve lead ECG. 


\section{Results}

We identified 137,835 patients from hospital A and after applying exclusion criteria, including 137,673 patients with 458,745 ECG for development dataset for developing ECGT2T. Among 137,673 patients from hospital A, 38,643 adult patients who underwent both $10 \mathrm{~s}$ 12-lead ECG and echocardiography within 14 days at hospital A were included to develop model to detect HFrEF using a 10 s 12-lead ECG. For external testing to confirm the performance for detecting HFrEF using smartwatch ECG, 761 patients from hospital B were identified and 6 patients were excluded due to missing values of ECG and echocardiography. Finally, 755 patients with 1510 ECG dataset (2-lead ECG from smart watch A and B) were included in the external performance test. As shown in Table 1, the HFrEF patients were older than the non-HFrEF patients. And HFrEF patients had more prolonged QRS interval and atrial fibrillation or flutter than the non-HFrEF patients.

Table 1. Baseline characteristics table.

\begin{tabular}{|c|c|c|c|c|c|c|c|c|c|}
\hline \multirow[b]{2}{*}{ Characteristics } & \multicolumn{4}{|c|}{$\begin{array}{c}\text { Hospital A (38,643 Patients) } \\
\text { Development and Interval Validation Data }\end{array}$} & \multicolumn{4}{|c|}{$\begin{array}{l}\text { Hospital B (755 Patients) } \\
\text { External Validation Smart Watch Data }\end{array}$} & \multirow[b]{2}{*}{$p \ddagger$} \\
\hline & HFrEF & HFmrEF & Normal & $p+$ & HFrEF & HFmrEF & Normal & $p+$ & \\
\hline Total Patients & $2519(6.5)$ & $1755(4.5)$ & $34,369(88.9)$ & & $39(5.2)$ & $31(4.1)$ & $685(90.7)$ & & 0.241 \\
\hline Age (year) & $64.82(13.52)$ & 64.98 (13.47) & $58.60(15.45)$ & $<0.001$ & $\begin{array}{c}60.69 \\
(13.84)\end{array}$ & $\begin{array}{c}58.97 \\
(14.46)\end{array}$ & $\begin{array}{c}55.61 \\
(15.16)\end{array}$ & 0.067 & $<0.001$ \\
\hline Male & 1665 (65.7) & $1083(61.3)$ & $16,341(47.6)$ & $<0.001$ & $29(74.4)$ & $20(64.5)$ & $325(47.4)$ & 0.001 & 0.969 \\
\hline Weight (Kg) & $64.68(13.90)$ & $65.25(13.34)$ & $64.87(12.60)$ & 0.341 & $\begin{array}{c}69.29 \\
(14.02)\end{array}$ & $\begin{array}{c}66.80 \\
(12.68)\end{array}$ & $\begin{array}{c}66.03 \\
(14.07)\end{array}$ & 0.358 & 0.004 \\
\hline Height (cm) & $162.68(9.56)$ & $\begin{array}{l}162.37 \\
(10.01)\end{array}$ & $162.20(9.58)$ & 0.044 & $\begin{array}{l}168.41 \\
(10.55)\end{array}$ & $\begin{array}{l}164.48 \\
(9.36)\end{array}$ & $\begin{array}{l}163.38 \\
(9.39)\end{array}$ & 0.005 & $<0.001$ \\
\hline $\begin{array}{l}\text { Body surface area } \\
\qquad\left(\mathrm{m}^{2}\right)\end{array}$ & $1.70(0.22)$ & $1.71(0.21)$ & $1.70(0.20)$ & 0.505 & $1.79(0.22)$ & $1.74(0.20)$ & $1.72(0.22)$ & 0.145 & 0.001 \\
\hline Heart rate (bpm) & $84.37(24.54)$ & $78.26(20.53)$ & $73.14(15.82)$ & $<0.001$ & $\begin{array}{c}78.31 \\
(19.78)\end{array}$ & $\begin{array}{c}70.03 \\
(14.55)\end{array}$ & $\begin{array}{c}69.95 \\
(12.56)\end{array}$ & 0.001 & $<0.001$ \\
\hline PR interval (ms) & $\begin{array}{l}175.83 \\
(36.69)\end{array}$ & $\begin{array}{l}176.83 \\
(37.36)\end{array}$ & $\begin{array}{l}167.99 \\
(29.01)\end{array}$ & $<0.001$ & $\begin{array}{l}122.00 \\
(60.53)\end{array}$ & $\begin{array}{c}156.74 \\
(144.40)\end{array}$ & $\begin{array}{l}149.46 \\
(97.07)\end{array}$ & 0.225 & $<0.001$ \\
\hline QRS duration (ms) & $\begin{array}{l}111.81 \\
(27.97)\end{array}$ & $\begin{array}{l}104.85 \\
(23.55)\end{array}$ & $95.47(15.88)$ & $<0.001$ & $\begin{array}{l}155.74 \\
(63.39)\end{array}$ & $\begin{array}{l}139.42 \\
(31.17)\end{array}$ & $\begin{array}{l}138.95 \\
(31.03)\end{array}$ & 0.010 & $<0.001$ \\
\hline QT interval (ms) & $\begin{array}{l}407.78 \\
(57.74)\end{array}$ & $\begin{array}{l}408.08 \\
(51.59)\end{array}$ & $\begin{array}{l}398.94 \\
(40.13)\end{array}$ & $<0.001$ & $\begin{array}{l}421.90 \\
(99.88)\end{array}$ & $\begin{array}{l}417.63 \\
(45.29)\end{array}$ & $\begin{array}{l}425.56 \\
(50.21)\end{array}$ & 0.681 & $<0.001$ \\
\hline $\begin{array}{l}\text { Atrial fibrillation of } \\
\text { flutter }\end{array}$ & $296(11.7)$ & $170(9.6)$ & $1172(3.4)$ & $<0.001$ & $3(7.7)$ & $1(3.2)$ & $14(2.0)$ & 0.076 & 0.015 \\
\hline P wave axis & 45.58 (39.72) & $44.48(35.84)$ & $45.32(28.89)$ & 0.585 & NA & NA & NA & NA & \\
\hline $\mathrm{R}$ wave axis & $27.71(65.00)$ & $31.15(53.61)$ & $39.80(42.07)$ & $<0.001$ & NA & NA & NA & NA & \\
\hline T wave axis & $83.07(85.26)$ & $58.82(72.34)$ & 42.57 (44.37) & $<0.001$ & NA & NA & NA & NA & \\
\hline $\begin{array}{c}\text { Ejection fraction } \\
(\%)\end{array}$ & $32.03(9.44)$ & $46.08(5.98)$ & $60.64(6.33)$ & $<0.001$ & $\begin{array}{l}31.23 \\
(7.21)\end{array}$ & $\begin{array}{l}45.97 \\
(2.50)\end{array}$ & $\begin{array}{l}64.63 \\
(5.19)\end{array}$ & $<0.001$ & $<0.001$ \\
\hline $\begin{array}{c}\text { Left atrial } \\
\text { dimension }(\mathrm{mm})\end{array}$ & 45.66 (8.97) & $44.05(9.48)$ & $38.98(7.84)$ & $<0.001$ & $\begin{array}{l}43.76 \\
(7.48)\end{array}$ & $\begin{array}{l}42.48 \\
(9.31)\end{array}$ & $\begin{array}{l}36.48 \\
(6.90)\end{array}$ & $<0.001$ & $<0.001$ \\
\hline 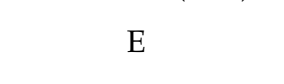 & $67.69(27.32)$ & $63.05(25.71)$ & $63.50(19.49)$ & $<0.001$ & $\begin{array}{l}72.00 \\
(22.11)\end{array}$ & $\begin{array}{c}68.65 \\
(27.26)\end{array}$ & $\begin{array}{c}66.55 \\
(19.21)\end{array}$ & 0.37 & $<0.001$ \\
\hline $\mathrm{A}$ & $68.40(23.50)$ & 71.03 (21.03) & $70.06(20.23)$ & 0.002 & $\begin{array}{c}71.28 \\
(22.05)\end{array}$ & $\begin{array}{c}74.00 \\
(25.71)\end{array}$ & $\begin{array}{c}66.74 \\
(20.64)\end{array}$ & 0.251 & $<0.001$ \\
\hline $\mathrm{E}^{\prime}$ & $5.04(1.91)$ & $5.72(2.09)$ & $7.10(2.67)$ & $<0.001$ & $5.80(3.81)$ & $6.06(2.54)$ & $7.64(4.62)$ & 0.044 & $<0.001$ \\
\hline $\mathrm{E} / \mathrm{E}^{\prime}$ & $14.90(7.84)$ & $12.04(6.27)$ & $9.88(4.58)$ & $<0.001$ & $\begin{array}{l}15.37 \\
(6.90)\end{array}$ & $\begin{array}{l}13.13 \\
(7.88)\end{array}$ & $9.85(4.22)$ & $<0.001$ & 0.534 \\
\hline
\end{tabular}

+ The alternative hypothesis for this $p$ value was that there was a difference between the heart failure with reduced ejection fraction, heart failure with mildly reduced ejection fraction, and non-heart failure. $\ddagger$ The alternative hypothesis for this $p$ value was that there is a difference between hospital A (derivation and internal validation data group) and hospital B (external validation group) for each variable. 
During internal validation of ECGs (hospital A), the AUC of the AI model to detect HFrEF and HFmrEF to HFrEF using a 10 s 12-lead ECG were 0.934 (0.929-0.938) and 0.909 (0.904-0.914), respectively. As shown in Figure 3, the ECGT2T generated a $10 \mathrm{~s}$ 12-lead ECG using a smartwatch 2-lead ECG (Lead I and II). We input the generated $10 \mathrm{~s}$ 12-lead ECG to develop an AI model that detects HFrEF using a 12-lead ECG. During external validation (hospital B), the AUC of AI for detecting HFrEF using ECG from smartwatch A and B were $0.946(0.925-0.968)$ and $0.925(0.888-0.963)$, respectively. The overall performance of 1510 ECG datasets (755 smartwatch A and 755 smartwatch B) was 0.934 (0.913-0.955), as shown in Figure 4. The AUC of ensemble score, defined as the average of the prediction scores of smartwatch A and B, was 0.954 (0.935-0.972). The sensitivity, specificity, PPV, and NPV of the AI model in smartwatch A were 0.974 (0.925-1.000), 0.821 (0.793-0.849), 0.229 (0.165-0.293), and 0.998 (0.995-1.000), respectively. The sensitivity, specificity, PPV, and NPV of the AI model in smartwatch B were 0.949 (0.879-1.000), 0.820 (0.792-0.848), 0.223 (0.160-0.286), and 0.997 (0.992-1.000), respectively. During external validation (hospital B) of secondary output, the AUC of AI for detecting HFmrEF to HFrEF using ECG from smartwatch A and B were 0.847 (0.795-0.898) and 0.845 (0.793-0.896), respectively.

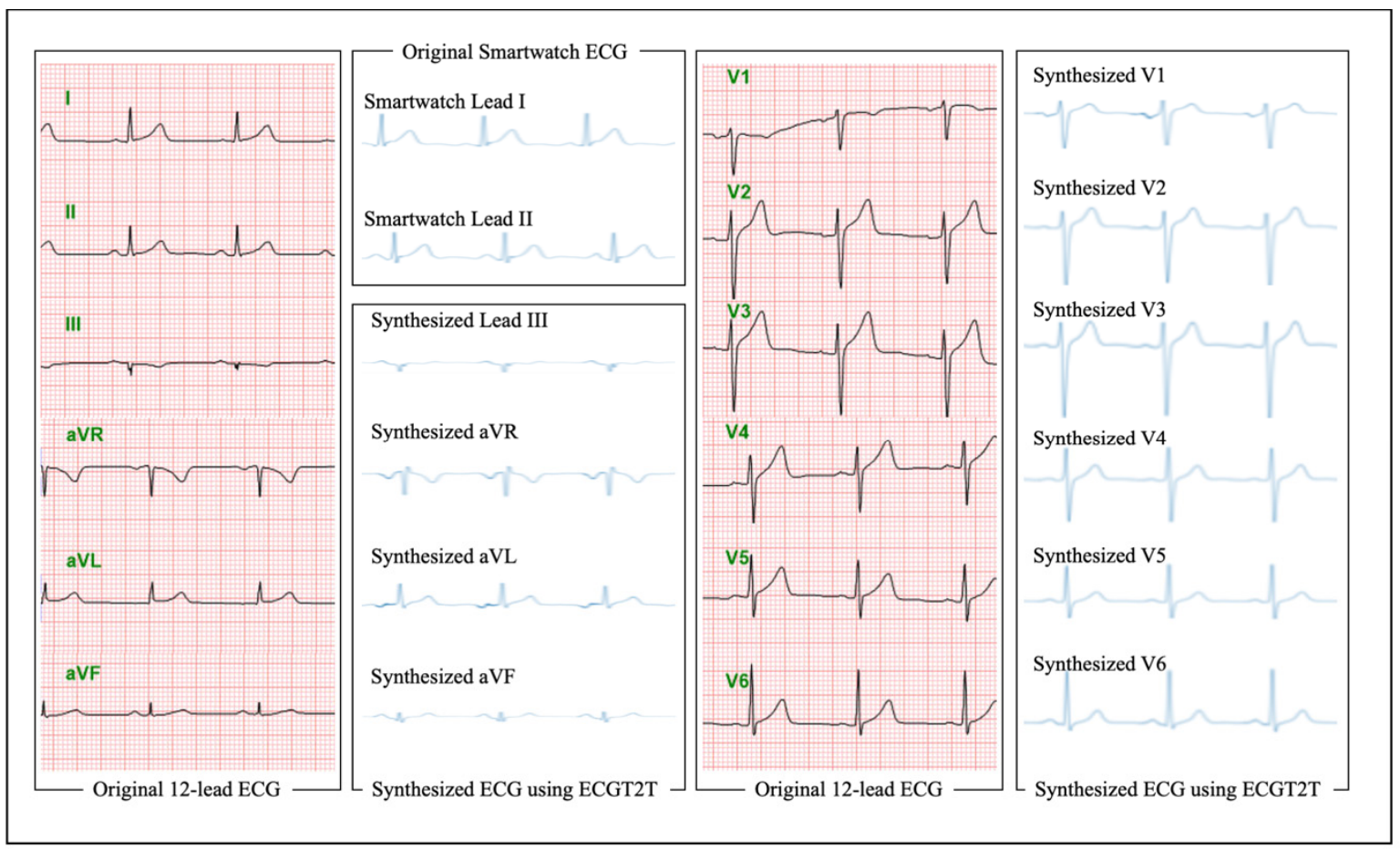

Figure 3. 12-lead ECG generation using smartwatch ECG based on ECGT2T. Legend: ECG denotes electrocardiography and ECGT2T ECG synthesis from two-lead to ten-lead. 


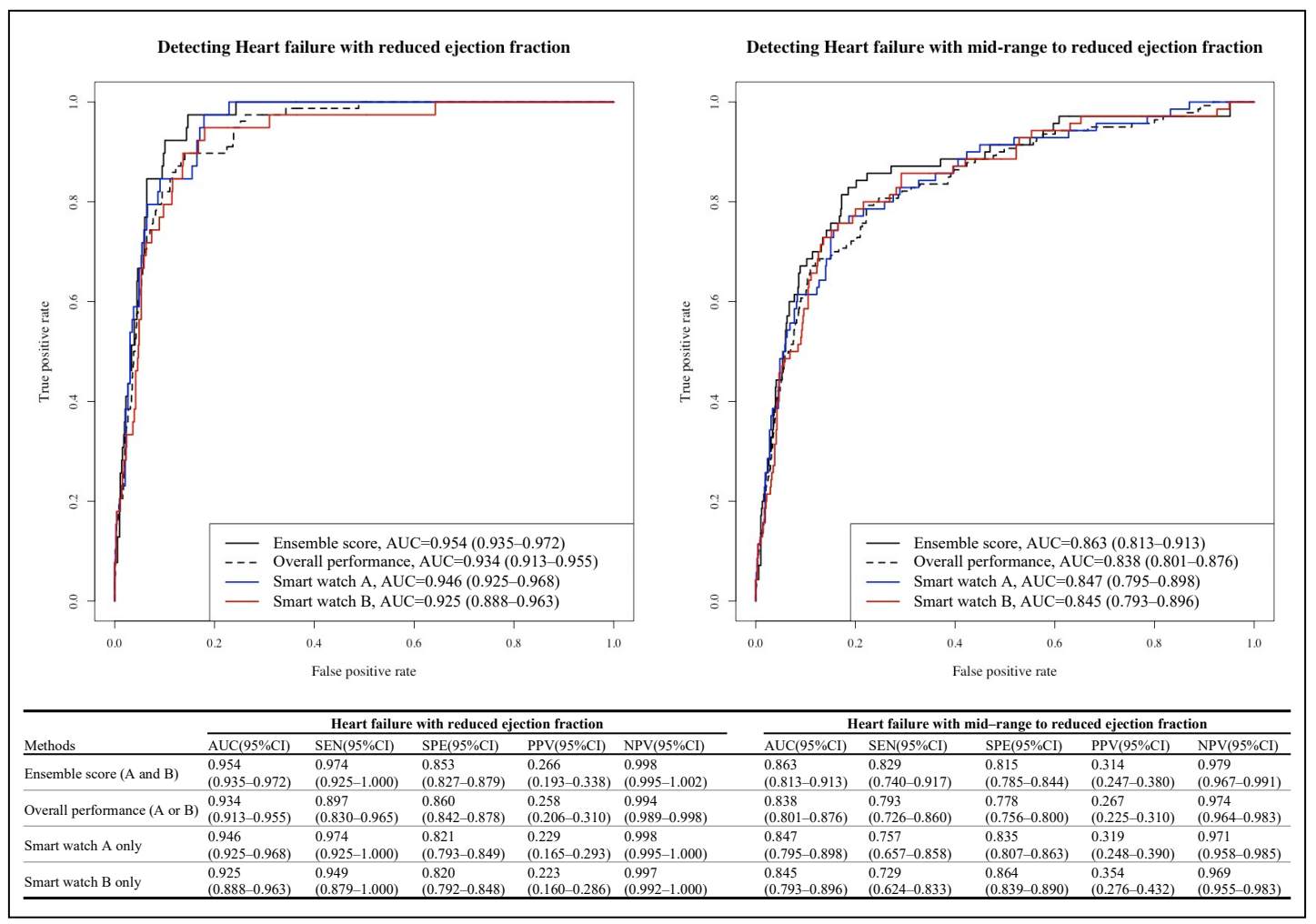

Figure 4. Performance of artificial intelligence for detecting heart failure using smart watch ECG. Legend: AUC denotes area under the receiver operating characteristic curve, CI confidence interval, ECG electrocardiography, NPV negative predictive value, PPV positive predictive value, SEN sensitivity, and SPE specificity.

\section{Discussion}

In this study, we proposed AI-enabled smartwatch ECG to detect HFrEF and it showed reasonable performance as a screening tool. These results outperformed other screening tools, such as B-type natriuretic peptide for HFrEF (AUC 0.871) [17]. This study showed the feasibility of using a smartwatch to diagnose diverse diseases other than arrhythmia. As this smartwatch is already used in our daily lives, we could monitor and detect HFrEF patients using our proposed AI model.

The ECG of each lead is a signal for measuring the electrical flow of the heart in each lead vector. The electrical vector of the heart can be estimated by synthesizing the ECG data of the two leads. A 12-lead ECG can be generated by reconstructing the ECG corresponding to the vector of each lead, determined based on the estimated electrical flow of the heart. ECGT2T is a deep learning model based on this concept and generates an ECG using a generative adversarial network.

Twelve-lead ECG was required for evaluating cardiovascular disease status using diverse vector information of the heart. However, in daily living, 12-lead ECG is not always practical or feasible because it is difficult to place the chest and limb lead at the exact location. In this study, we generated 12-lead ECG and detected heart failure with reduced ejection fraction using only leads I and II, which could be captured by a smart watch in use in daily living. Therefore, this study is a milestone in using ECG to detect cardiovascular disease in daily living. Although previous studies have detected diseases using ECG, studies using 12-lead ECG and other devices have been conducted only in hospital settings. Here, we developed a deep learning methodology that generates ECGs from lifestyle devices and smartwatches. Therefore, our methodology can be used in daily living. The major contribution of this study is to provide a methodology for detecting 
disease in daily living based on deep learning model using a single lead life style device, such as a smart watch.

The increasing prevalence of HF making it among the most costly diseases to Medicare [18]. More than $30 \%$ HF patients are seen in the clinic setting, and more than $40 \%$ of those recently admitted with decompensation will require a second hospitalization within a year [19]. Early detection of HFrEF offers the opportunity to test and develop an effective lifestyle and life-saving medical therapy [9]. The evolution and adoption of digital health technology and mobile health devices may address this issue. Our day-to-day lives are impacted by technological innovations, and the recent trend of commercial smart wearable devices aims in improving our health [20]. Smart wearables are connected electronic devices designed for everyday use that can be worn on the body as an accessory or integrated into clothing. Smartwatches and wristbands have high processing power and sophisticated sensors that can provide new health information [20]. Wearable health devices are an aspect of medical health that may improve the delivery of HF care by allowing medical data collection outside of a clinician's office or hospital. Wearable devices are externally applied and capture functional or physiological data to monitor and improve patients' health. It could be a cost-effective method to detect HF before it becomes fatal. Personalized patient care has become remote and decentralized owing to the COVID-19 pandemic [21]. The cardiovascular community must utilize the commercially available wearable technologies as well as the wide range of clinical applications that they can serve. This technology integration into the clinical workplace, however, is still in its early stages.

Per previous medical knowledge, ECGs could be used to detect small coverage diseases, such as arrhythmia and ST-segment elevation myocardial infarction. We could not develop diagnostic criteria and tools using non-linear correlations between diverse diseases and subtle changes based on conventional statistical methods, such as logistic regression [22]. Recently, AI has been adopted to diagnose many diseases and conditions and to predict the development of disease [23]. Most AI for detecting diseases using ECG is based on deep learning. The most important strength of deep learning is the automatic feature extraction [24]. Specifically, deep learning automatically extracts the features of ECG to detect disease, without any human engineering resource needed to define the features for using the model. This has reduced the time and cost of AI development. The importance of automatic feature extraction is that we can extract features and develop a model without human prejudice. AI based on deep learning is based only on information from data, not medical knowledge. This aspect showed the possibility of enhancing the model to detect diverse diseases and show new medical findings over previous medical knowledge.

An important pitfall of deep learning is overfitting [23]. Especially, deep learning can be subjugated to the environment in which development data are obtained. Therefore, we should validate the developed AI to other hospitals and environments. The important point of this study was external validation. We confirmed the performance of the AI model using data from other hospitals and other devices in this study. In other word, we developed AI using 12-lead ECG data from hospital A and external validated AI using smartwatch ECG from hospital B.

The advantage of AI is that it saves healthcare costs. Using this AI, HFrEF could be screened by a wearable watch without a physician, and it could refer patients at risk to cardiologists for confirmative diagnostic tests. Therefore, this procedure is advantageous for low-income countries to save patients from irreversible disease progression and death This AI could be used in wearable watches in daily living and HFrEF could be detected and monitored in the early stages without complications. This predictive care solution is essential to reduce healthcare costs.

This study had several limitations. First, we validated the AI-enabled smartwatch ECG to detect HFrEF in a hospital setting. As there is a possibility of decreasing performance in daily living at home, we needed to validate this AI in a home setting [25]. Second, this study was conducted in South Korea, and it is necessary to validate the AI in other 
countries. We will verify the performance and significance of the proposed model through a prospective remote home care setting in a multinational study.

Author Contributions: J.-m.K. contributed to the study idea and design and data analysis, developed the deep learning model, verified the clinical coding, and contributed to the subsequent draft. Y.Y.J. developed the backbone of the deep learning model and performed the data analysis. M.S.L. contributed to the performed data analysis and contributed to the subsequent draft. S.Y.L. contributed to the study idea and design and subsequent draft. S.K. and S.-Y.L. performed the data analysis and verified the clinical coding. K.-H.K. is the principal investigator and contributed to the study idea and design, data analysis, and subsequent drafts. All authors have read and agreed to the published version of the manuscript.

Funding: This work was supported by a National Research Foundation of Korea (NRF) grant funded by the Korea government (MSIT) (No. 2020R1F1A1073791).

Institutional Review Board Statement: The study was conducted according to the guidelines of the Declaration of Helsinki, and approved by the Institutional Review Board of Incheon Sejong Hospital (protocol code ISH 2021-05-010-002 and date of approval 20 October 2021).

Informed Consent Statement: Patient consent was waived due to retrospective nature and minimal harm.

Data Availability Statement: The data underlying this article will be shared on reasonable request to the corresponding author.

Acknowledgments: This research was results of a study on the "Healthcare Data Center Hospitals" Project, supported by the Ministry of Health and Welfare of South Korea and Korea Health Information Service.

Conflicts of Interest: K.-H.K. and S.Y.L. declare that they have no competing interests. J.-m.K. is co-founder and Y.-Y.J., MS Lee, S K., and S.-Y.L. are researchers of Medical AI Inc., a medical artificial intelligence company. J.-m.K. is a researcher of Body friend Co., Ltd. There are no products in development or marketed products to declare. This does not alter our adherence to this journal.

\section{References}

1. GBD 2017 Disease and Injury Incidence and Prevalence Collaborators. Global, Regional, and National Incidence, Prevalence, and Years Lived with Disability for 354 Diseases and Injuries for 195 Countries and Territories, 1990-2017: A Systematic Analysis for the Global Burden of Disease Study. Lancet 2018, 392, 1789-1858. Available online: http:/ /www.ncbi.nlm.nih.gov/pubmed/30496104 (accessed on 8 October 2021). [CrossRef]

2. Lam, C.S.P.; Gamble, G.D.; Ling, L.H.; Sim, D.; Leong, K.T.G.; Yeo, P.S.D.; Ong, H.Y.; Jaufeerally, F.; Ng, T.P.; Cameron, V.A.; et al. Mortality associated with heart failure with preserved vs. reduced ejection fraction in a prospective international multi-ethnic cohort study. Eur. Heart J. 2018, 39, 1770-1780. Available online: http://www.ncbi.nlm.nih.gov/pubmed/29390051 (accessed on 8 October 2021). [CrossRef] [PubMed]

3. Virani, S.S.; Alonso, A.; Benjamin, E.J.; Bittencourt, M.S.; Callaway, C.W.; Carson, A.P.; Chamberlain, A.M.; Chang, A.R.; Cheng, S.; Delling, F.N.; et al. American Heart Association Council on Epidemiology and Prevention Statistics Committee and Stroke Statistics Subcommittee. Heart Disease and Stroke Statistics-2020 Update: A Report from the American Heart Association. Circulation 2020, 141, e139-e596. Available online: http:/ /www.ncbi.nlm.nih.gov/pubmed/31992061 (accessed on 8 October 2021). [CrossRef] [PubMed]

4. Braunwald, E. The war against heart failure: The Lancet lecture. Lancet 2015, 385, 812-824. [CrossRef]

5. Groenewegen, A.; Rutten, F.H.; Mosterd, A.; Hoes, A.W. Epidemiology of heart failure. Eur. J. Heart Fail. 2020, $22,1342-1356$. Available online: http:/ / www.ncbi.nlm.nih.gov/pubmed/32483830 (accessed on 8 October 2021). [CrossRef] [PubMed]

6. Savarese, G.; Lund, L.H. Global Public Health Burden of Heart Failure. Card. Fail. Rev. 2017, 3, 7-11. [CrossRef]

7. Flint, K.M.; Fairclough, D.L.; Spertus, J.A.; Bekelman, D.B. Does heart failure-specific health status identify patients with bothersome symptoms, depression, anxiety, and/or poorer spiritual well-being? Eur. Hear. J. Qual. Care Clin. Outcomes 2019, 5, 233-241. [CrossRef]

8. Murphy, S.P.; Ibrahim, N.E.; Januzzi, J.L. Heart Failure with Reduced Ejection Fraction. JAMA 2020, 324, 488-504. Available online: https://jamanetwork.com/journals/jama/fullarticle/2768982 (accessed on 8 October 2021). [CrossRef]

9. McDonagh, T.A.; Metra, M.; Adamo, M.; Gardner, R.S.; Baumbach, A.; Böhm, M.; Burri, H.; Butler, J.; Čelutkienė, J.; Chioncel, O.; et al. 2021 ESC Guidelines for the diagnosis and treatment of acute and chronic heart failure. Eur. Heart J. 2021, 42, 3599-3726. Available online: https://academic.oup.com/eurheartj/advance-article/doi/10.1093/eurheartj/ehab368/6358045 (accessed on 8 October 2021). [CrossRef] 
10. Jonas, D.E.; Reddy, S.; Middleton, J.C.; Barclay, C.; Green, J.; Baker, C.; Asher, G.N. Screening for Cardiovascular Disease Risk with Resting or Exercise Electrocardiography. JAMA 2018, 319, 2315-2328. [CrossRef]

11. Kwon, J.-M.; Kim, K.-H.; Jeon, K.-H.; Kim, H.M.; Kim, M.J.; Lim, S.-M.; Song, P.S.; Park, J.; Choi, R.K.; Oh, B.-H. Development and Validation of Deep-Learning Algorithm for Electrocardiography-Based Heart Failure Identification. Korean Circ. J. 2019, 49, 629-639. [CrossRef] [PubMed]

12. Cho, J.; Lee, B.; Kwon, J.-M.; Lee, Y.; Park, H.; Oh, B.-H.; Jeon, K.-H.; Park, J.; Kim, K.-H. Artificial Intelligence Algorithm for Screening Heart Failure with Reduced Ejection Fraction Using Electrocardiography. ASAIO J. 2020, 67, 314-321. [CrossRef] [PubMed]

13. Spaccarotella, C.A.M.; Polimeni, A.; Migliarino, S.; Principe, E.; Curcio, A.; Mongiardo, A.; Sorrentino, S.; De Rosa, S.; Indolfi, C. Multichannel Electrocardiograms Obtained by a Smartwatch for the Diagnosis of ST-Segment Changes. JAMA Cardiol. 2020, 5, 1176-1180. [CrossRef] [PubMed]

14. Bozkurt, B.; Coats, A.J.; Tsutsui, H.; Abdelhamid, M.; Adamopoulos, S.; Albert, N.; Anker, S.D.; Atherton, J.; Böhm, M.; Butler, J.; et al. Universal Definition and Classification of Heart Failure. J. Card. Fail. 2021, 27, 387-413. Available online: https:/ / linkinghub.elsevier.com/retrieve/pii/S1071916421000506 (accessed on 8 October 2021). [CrossRef] [PubMed]

15. Jo, Y.-Y.; Kwon, J.-M. Electrocardiogram synthesis from two asynchronoous leads to Ten leads. arXiv 2021, arXiv:2103.00006. Available online: http:/ / arxiv.org/abs/2103.00006 (accessed on 8 October 2021).

16. Schisterman, E.F.; Perkins, N.J.; Liu, A.; Bondell, H. Optimal Cut-point and Its Corresponding Youden Index to Discriminate Individuals Using Pooled Blood Samples. Epidemiology 2005, 16, 73-81. [CrossRef] [PubMed]

17. Lokuge, A.; Lam, L.; Cameron, P.; Krum, H.; Smit, D.V.; Bystrzycki, A.; Naughton, M.T.; Eccleston, D.; Flannery, G.; Federman, J.; et al. B-Type Natriuretic Peptide Testing and the Accuracy of Heart Failure Diagnosis in the Emergency Department. Circ. Hear. Fail. 2010, 3, 104-110. [CrossRef]

18. Jackson, S.L.; Tong, X.; King, R.J.; Loustalot, F.; Hong, Y.; Ritchey, M.D. National Burden of Heart Failure Events in the United States, 2006 to Circ. Hear. Fail. 2018, 11, e004873. [CrossRef]

19. Cowie, M.R.; Anker, S.D.; Cleland, J.G.F.; Felker, G.M.; Filippatos, G.; Jaarsma, T.; Jourdain, P.; Knight, E.; Massie, B.; Ponikowski, P.; et al. Improving care for patients with acute heart failure: Before, during and after hospitalization. ESC Hear. Fail. 2014, 1, 110-145. [CrossRef]

20. Perez, M.V.; Mahaffey, K.W.; Hedlin, H.; Rumsfeld, J.S.; Garcia, A.; Ferris, T.; Balasubramanian, V.; Russo, A.M.; Rajmane, A.; Cheung, L.; et al. Large-Scale Assessment of a Smartwatch to Identify Atrial Fibrillation. N. Engl. J. Med. 2019, 381, $1909-1917$. [CrossRef]

21. Mishra, T.; Wang, M.; Metwally, A.A.; Bogu, G.K.; Brooks, A.W.; Bahmani, A.; Alavi, A.; Celli, A.; Higgs, E.; Dagan-Rosenfeld, O.; et al. Pre-symptomatic detection of COVID-19 from smartwatch data. Nat. Biomed. Eng. 2020, 4, 1208-1220. [CrossRef] [PubMed]

22. Breiman, L. Statistical Modeling: The Two Cultures. Stat. Sci. 2001, 16, 199-215. [CrossRef]

23. Kwon, J.-M.; Jo, Y.-Y.; Lee, S.Y.; Kim, K.-H. Artificial intelligence using electrocardiography: Strengths and pitfalls. Eur. Hear. J. 2021, 42, 2896-2898. [CrossRef] [PubMed]

24. LeCun, Y.; Bengio, Y.; Hinton, G. Deep learning. Nature 2015, 521, 436-444. Available online: http://arxiv.org/abs /1603.05691 (accessed on 8 October 2021). [CrossRef]

25. Tison, G.; Sanchez, J.M.; Ballinger, B.; Singh, A.; Olgin, J.E.; Pletcher, M.J.; Vittinghoff, E.; Lee, E.S.; Fan, S.M.; Gladstone, R.A.; et al. Passive Detection of Atrial Fibrillation Using a Commercially Available Smartwatch. JAMA Cardiol. 2018, 3, 409-416. [CrossRef] 